\title{
Impact of antidepressant treatment: a study from The Netherlands
}

This article was published in the following Dove Press journal:

Patient Intelligence

17 July 2012

Number of times this article has been viewed

\author{
Nadine van Dongen' \\ Scott Mason ${ }^{2}$ \\ 'PIP Health Ltd, London, United \\ Kingdom; ${ }^{2}$ London Metropolitan \\ University, London, United Kingdom
}

Background: The purpose of the study was to identify the extent and severity of treatmentrelated side effects experienced by patients with depression. The study was designed to identify the extent of the different antidepressant side effects to gain an understanding of their impact on the lives of patients suffering from depression.

Methods: A total of 303 patients from The Netherlands with physician-diagnosed depression completed an online questionnaire. All participating patients were receiving antidepressants and were members of the Patient Intelligence Panel (PIP). Participants answered questions about their antidepressant medication, degree of personal research into antidepressant medication, and about any side effects they had experienced as a result of their current antidepressant therapy, the severity of any such side effects, and the impact on their daily lives.

Results: Over two-thirds (69\%) of patients were receiving antidepressant therapy for the first time. Forty-two percent ( $42 \%$ ) of patients felt it took 2-6 weeks of antidepressant therapy before their depressive mood improved. More than half of participating patients $(60 \%)$ had experienced side effects from their antidepressant therapy, the most common being 'somnolence, drowsiness, or fatigue' (14\%). Of the $60 \%$ of patients who had experienced side effects, $46 \%$ admitted to absenteeism as a consequence.

Conclusion: Central to the effective management of depression is an understanding of the effects of antidepressant therapy on patients' lives. Treatment-related side effects, and their potential influence on treatment discontinuation, need to be recognized and assessed on an individual patient basis to optimize management and minimize potential relapse.

Keywords: depression, antidepressants, SSRI, side effects, The Netherlands

\section{Introduction}

The World Health Organization (WHO) estimates that 121 million people suffer from depression worldwide. It is a condition that occurs in people of all ages, sexes, nationalities, and socioeconomic backgrounds. At its most severe, depression can lead to suicide and is estimated to be responsible for 850,000 deaths every year. ${ }^{1}$ In terms of mortality and disability, major depression is the leading cause of premature death and ongoing disability. ${ }^{2}$ Data from the WHO's World Mental Health Survey Initiative suggests $15 \%$ of people from high-income countries are likely to suffer from depression at some point during their lifetime; the prevalence among women is twice that of men. ${ }^{3}$

In terms of management and diagnosis, depression is most reliably diagnosed in primary care. Antidepressant medications and psychotherapy, which form the backbone of therapeutic management, are effective in $60 \%-80 \%$ of patients
PIP Health Ltd, 77 Bastwick Street, London ECIV 3PZ, United Kingdom Email nadine@piphealth.com 
managed in primary care. While questions persist about the relative effectiveness of different antidepressant treatments, the overriding benefits of pharmacological and psychological therapies are evident in terms of improved engagement with health care, reduced stigmatization of affected patients, and reduced suicide rates, absenteeism, alcohol and substance misuse, and cardiovascular disease risk. ${ }^{4-6}$

Despite the benefits of antidepressant therapies, there are side effects associated with their use. The extent of antidepressant side effects is associated with their site of action and receptor selectivity. The safety and tolerability profiles of newer antidepressants (eg, selective serotonin reuptake inhibitors [SSRIs] and serotonin-norepinephrine reuptake inhibitors [SNRIs]) are better than those of the more traditional antidepressants because of their selectivity for serotonin receptors. Yet side effects exist even with these more selective antidepressants and clinical experience suggests that they are more frequent than was suggested by the initial randomized controlled trial (RCT) data. ${ }^{7}$

SSRIs were introduced to the United States in the late 1980s, marking a significant advance in the management of depression. Although comparable to the older monoamine oxidase inhibitors (MAOIs) and tricyclic antidepressants (TCAs) in terms of efficacy, they were associated with reduced side effects and lower rates of treatment discontinuation than TCAs. ${ }^{8}{ }^{8}$ Moreover, SSRIs had a lower propensity to cause seizures than TCAs and were not associated with cardiac conduction abnormalities. ${ }^{10}$

This study was designed to evaluate the nature and extent of the side effects experienced by a cohort of patients from The Netherlands diagnosed with depression in 2009 and managed on licensed antidepressant therapies. The aim of the study was to gain a better understanding of the true burden of licensed antidepressant treatments. The type and severity of treatment-related side effects were assessed, as was the associated impact on the daily lives of affected patients.

\section{Methodology}

This was an online questionnaire-based study of adult patients (aged 18-65 years) from The Netherlands. The study was conducted in 2009 and included patients with physiciandiagnosed depression who were actively managed on licensed antidepressant medications.

Patient Intelligence Panel (PIP) Health developed the questionnaire, in collaboration with the authors of this paper. PIP Health also identified eligible patients and programmed and fielded the study. The sampling plan was designed to generate a study population representative, in terms of age and sex, of the true population of patients in the Netherlands living with diagnosed depression (as defined by 2009 national statistics from the Dutch Bureau of National Statistics [CBS]).

To be eligible for inclusion, patients had to be aged 18-65 years, have self-reported physician-diagnosed depression and be receiving antidepressant therapy at the time of the study, or in the preceding 12 months. Patients were excluded if they had been hospitalized due to depression, or had not received antidepressant therapy in the last 12 months.

Invited participants were members of PIP Health's online patient panel who, at the time of panel enrolment, indicated that they had depression. An invitation email was sent through PIP Health's online database to 844 patients with self-reported physician-diagnosed depression. Potential study participants received an email message inviting them to fill out an online questionnaire that would take approximately 10 minutes to complete. The email included a link to the online questionnaire and offered patients a $€ 1$ charitable donation to the Dutch patient adherence group 'Korrelatie' as an incentive. The invitation clearly stated that the survey was designed in accordance with the Association of the British Pharmaceutical Industry (ABPI), Market Research (MRS), and British Healthcare Business Intelligence Association (BHBIA) codes of conduct regarding patient anonymity and confidentiality. Of the 844 patients invited, 354 completed the questionnaire and 303 were included in the reported analysis; 51 respondents (14\%) were excluded from the analysis through failure to meet the full study eligibility criteria.

\section{Measures}

\section{Perceived side effects - incidence and severity}

The questionnaire was designed to explore the side effects associated with physician-prescribed antidepressant therapies. Patients were first asked to indicate ('Yes/No') if they had experienced side effects from antidepressant therapy. Patients who had experienced side effects were then asked to indicate which side effects, from a list of 14 possibilities, they had experienced; more than one item on the list could be selected. The list of possible side effects was taken from a study conducted by Van Geffen that evaluated patients' experiences with antidepressants using a medicine reporting system. Side effects were coded by one of the authors (Van Der Wal) using the World Health Organization Adverse Reaction Terminology (WHO-ART) and checked and verified by a second author (Van Geffen). ${ }^{11,12}$ If the side effects experienced by patients in this study were not included 
on the Van Der Wal list of possibilities, there was an option to select 'other' and note the nature of the side effects they had experienced.

Patients were also asked to grade the severity of their side effects on a Likert scale of 1 ('not severe') to 10 ('very severe') to allow an exploration of (i) the impact of antidepressant side effects on patients' daily lives and (ii) an association between severity and workplace absenteeism.

\section{Perception of effects of side effects on daily living - absenteeism}

The questionnaire was designed to investigate the impact of antidepressant side effects on patients' lives. Those respondents who had experienced antidepressant side effects were asked to indicate ('Yes/No') whether they had ever taken time off work because of the side effects. They were then asked to give examples (in note format) of the ways in which they felt treatment side effects had affected their daily lives. The open-ended answers enabled patients to focus on the specific issues most important to them.

\section{Perceived onset of action}

In addition to assessing the extent and impact of the side effects associated with antidepressant therapy, patients were asked to indicate how long they felt it took between initiating antidepressant therapy and experiencing an improvement in their depressive symptoms. They had to select one of four options: 'between 0-2 weeks'; 'between 2-6 weeks'; 'more than 6 weeks'; or 'symptoms never improve'. Patient responses were split by therapy type and averaged to indicate the relative effectiveness of the antidepressants available in The Netherlands at the time of the study.

\section{Results}

A total of 303 eligible patients took part in the study. More females than males participated ( $77 \%$ vs $23 \%$, respectively) and the most common age category was $41-45$ years old. The average level of education (in line with the Dutch educational system) was MBO, ie, the equivalent of English A-levels (see Table 1).

Eighty-six percent of patients had been diagnosed with depression by their general practitioner (GP) and were being managed in primary care; $9 \%$ were diagnosed and being treated by a health care professional other than a GP (eg, a psychiatrist). The remaining $5 \%$ of patients answered 'other' to this question.

More than two-thirds of patients (69\%) were being treated with an antidepressant for the first time. The remaining
Table I Educational level of participating patients

\begin{tabular}{lll}
\hline & Frequency & Percent \\
\hline Primary school & 8 & $3 \%$ \\
Secondary school & 79 & $26 \%$ \\
A-level & 116 & $38 \%$ \\
University BA/BSc & 80 & $26 \%$ \\
Masters & 20 & $7 \%$ \\
Total & 303 & $100 \%$ \\
\hline
\end{tabular}

Abbreviations: BA, Bachelor of Arts; BSc, Bachelor of Science.

$31 \%$ of patients had used more than one type of antidepressant at some point, the majority of whom had used two antidepressant treatments. The distribution of antidepressant drugs (type/category) being used by the study population is summarized in Table 2.

\section{Perceived side effects - incidence and severity}

More than half of participating patients (60\%) had experienced some degree of side effects while using antidepressants. When asked to specify the nature of the side effects they had experienced, the most commonly reported side effects were 'somnolence, drowsiness, or fatigue,' 'weight increase,' and 'sexual problems,' experienced by $14 \%, 13 \%$, and $12 \%$ of patients, respectively. Among those patients experiencing antidepressant side effects, the average number of side effects reported per patient was 3.8. The types and frequency of side effects experienced by the study population are summarized in Figure 1.

In terms of treatment severity, the average severity rating across all side effects (on a Likert scale of $1=$ 'not severe' to $10=$ 'very severe') was 7.0. Figure 2 details the mean severity rating for each reported side effect; all mean severity

Table 2 Distribution of antidepressant prescribing within the study population

\begin{tabular}{lll}
\hline Antidepressant & Drug class & Percent of patients \\
\hline Paroxetine & SSRI & $30 \%$ \\
Fluoxetine & SSRI & $16 \%$ \\
Venlafaxine & SNRI & $10 \%$ \\
Citalopram & SSRI & $7 \%$ \\
Amitriptyline & TCA & $7 \%$ \\
Mirtazapine & NaSSA & $7 \%$ \\
Sertraline & SSRI & $7 \%$ \\
Fluvoxamine & SSRI & $6 \%$ \\
Clomipramine & TCA & $4 \%$ \\
Other & - & $3 \%$ \\
Escitalopram & SSRI & $2 \%$ \\
Duloxetine & SNRI & $1 \%$ \\
\hline
\end{tabular}

Abbreviations: SSRI, selective serotonin reuptake inhibitor; SNRI, serotoninnorepinephrine reuptake inhibitor; TCA, tricyclic antidepressant; NaSSA, noradrenergic and specific serotonergic antidepressant. 


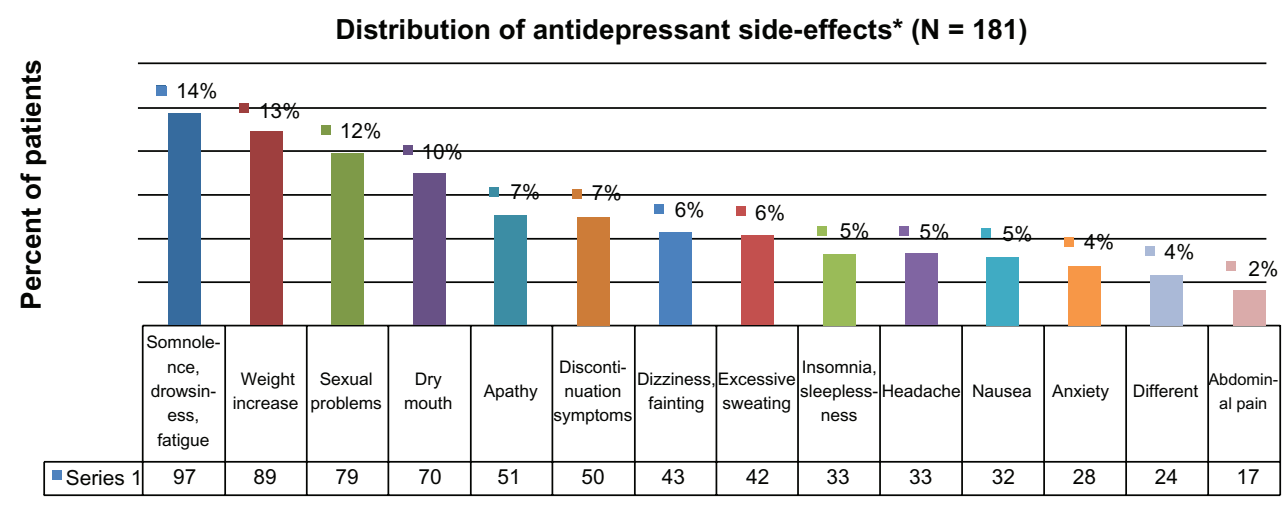

Figure I Distribution of side effects associated with antidepressant medication (among those patients reporting some degree of treatment-related side effects, $\mathrm{N}=\mathbf{I 8 I}$ ). Note: *English answers; see survey for Dutch translations.

scores were toward the more severe end of the rating scale, ie, $>5$. The most severe side effect, rated at 8.16 , was 'other,' reflecting the presence of additional severe side effects that patients felt the need to express in the free-text response field. Table 3 summarizes all 'other' side effects reported by study participants and illustrates the wide variety of side effects that the patients with depression can experience on antidepressant medication. No severe adverse events requiring reporting were recorded through the 'other' side effects free text option.

The most severe of side effects on the Van der Wal list were 'anxiety' and 'insomnia' (rated 7.53 and 7.45, respectively); dry mouth was the least severe, rated an average severity of 6.22 (see Figure 2). The three most commonly reported side effects ('somnolence, drowsiness, fatigue,' 'weight increase,' and 'sexual problems') had relatively high average severity scores: 6.87, 6.93, and 7.1, respectively.

Discontinuation of antidepressant therapy can be challenging, with some patients restarting therapy just days after ceasing their previous therapy. 'Discontinuation problems' was rated an average score of 7.06 , and almost one-third of patients $(31 \%)$ had used more than one antidepressant at some point in the past.

\section{Perception of effects of side effects on daily living - absenteeism}

Almost half (46\%) of the 181 patients who had experienced some form of antidepressant side effects had missed work as a result. When asked to provide more information about the extent to which side effects affected their daily lives, patients provided a number of examples in their notes of how they felt their antidepressants had impaired their quality of life and/or impeded their ability to work effectively. A number of examples of these lifestyle limitations are detailed in Table 4, illustrating the real-life impact of antidepressants on the daily lives of patients with depression.

\section{Perceived onset of action}

The Dutch College of General Practitioners (NHG) recommends antidepressant medication be continued for at least 4-6 weeks as it can take 2-6 weeks for patients to feel the benefit of treatment. ${ }^{13}$ This was reflected by the study population with 2-6 weeks being the average time between

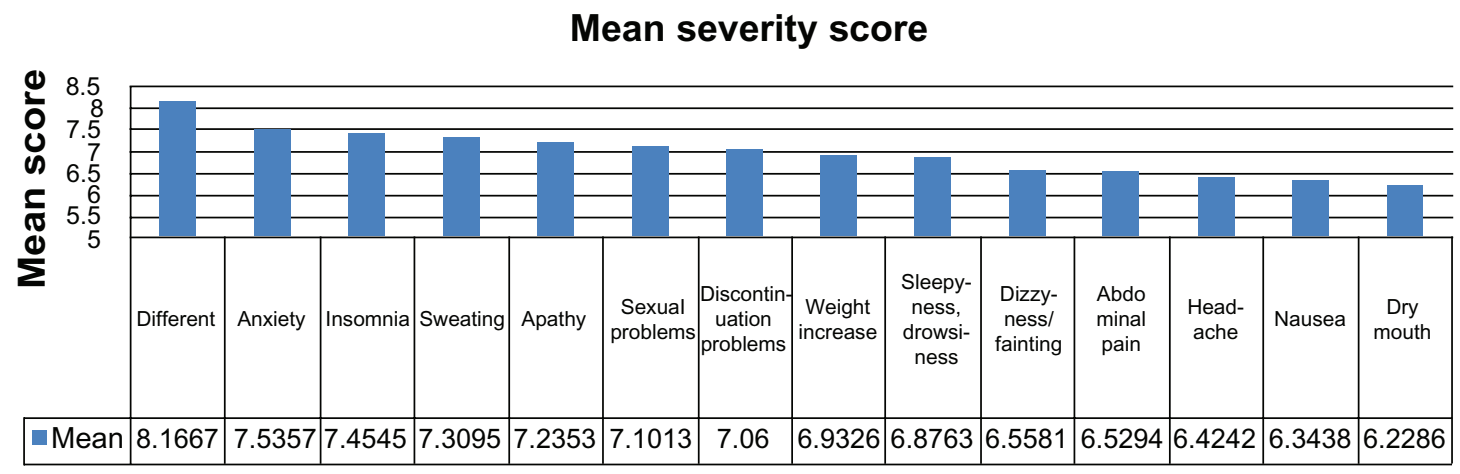

Figure 2 Mean side-effect severity scores. 
Table 3 Other antidepressant side effects reported by patients (notes)

Patient-reported side effects
Black outs
Diarrhea
Disturbed heart rhythm
Extreme stress/pressure
High tone in ear
Hypertension
Itching
Living like a zombie
Weight loss
Loss of concentration
Lowering blood pressure
Inability to express oneself
Bladder pressure
Shaking
Shaking hands
Skin irritation
Stiff jaw
Thick tongue
Uncontrollable bladder
Uncontrollable tensions
Restless leg syndrome
Poor eyesight

patients starting antidepressant therapy and them perceiving a beneficial effect of treatment. Within the study population, $21 \%$ of patients felt it had taken less than 2 weeks for their antidepressant medication to take effect; $42 \%$ that it had taken 2-6 weeks; $24 \%$ more than 6 weeks; and $13 \%$ of patients felt that their antidepressant therapy led to no improvement in their depression (see Table 5).

Table 4 Examples of the ways in which participating patients felt antidepressant side effects had affected and impaired their daily lives (notes)

\begin{tabular}{l}
\hline Impact of antidepressants on daily functioning \\
\hline I'm too tired to work \\
I'm tired during the day. At night I cannot sleep so I am too tired to work \\
I'm much too tired to go to work \\
I had to stop working due to excessive sweating; not very hygienic when \\
you work in a kitchen \\
I felt sick and had stomach acid and headaches that made working full \\
time impossible \\
Postnatal depression, could not work, was drowsy and tired \\
so couldn't work \\
Sleepy during work \\
Felt very dizzy at work, felt like there was electricity in my head! \\
I didn't feel emotions anymore, no interest for anything and dangerous \\
to drive to work so I could not go \\
Too dizzy to work \\
After work during the day I went to sleep \\
I was so tired I could not go to work \\
Less active both at home and at work
\end{tabular}

Table 5 Duration of antidepressant therapy before patientperceived benefit

\begin{tabular}{lll}
\hline & Frequency & Percent \\
\hline Never & 41 & $13 \%$ \\
Between 0-2 weeks & 62 & $21 \%$ \\
Between 2-6 weeks & 126 & $42 \%$ \\
More than 6 weeks & 74 & $24 \%$ \\
Total & 303 & $100 \%$ \\
\hline
\end{tabular}

\section{Discussion}

This study found that, on average, patients experience an improvement in their depression after 2-6 weeks of antidepressant therapy. Although this was the most frequently cited time to perceived treatment benefit, a substantial proportion of patients in this Dutch cohort (13\%) felt that their antidepressant therapy had had no positive effect on improving their depression.

Overall, $87 \%$ of patients reported some benefit of antidepressant therapy, which is significantly higher than the $30 \%-45 \%$ quoted in a recent study and in the lay press in recent years. ${ }^{14}$ Indeed, in 2002 the New York Times published an article entitled Antidepressants Lift Clouds, But Lose 'Miracle Drug' Label, claiming only 35\%-45\% of patients with depression benefit from antidepressant therapy. Although a high proportion of patients in this study indicated some degree of treatment benefit, the proportions who felt their treatment 'never' worked $(13 \%)$ or took 'more than 6 weeks' to take effect (24\%) suggest that licensed antidepressant therapies fail to satisfy the expectations of a substantial number of real-life patients.

When SSRIs were first introduced, they were considered to be almost free of side effects and suitable for use by a wide variety of patients, particularly those susceptible to side effects on TCAs. As a consequence of their overall safety, efficacy, and tolerability profiles, they are widely prescribed by primary care physicians. ${ }^{7}$ In this study, $82 \%$ of patients were prescribed SSRIs/SNRIs (see Table 2). The 11\% of patients prescribed TCAs and 7\% noradrenergic and specific serotonergic antidepressants (NaSSAs) were too small to have a significant impact on the study results.

Although SSRIs are recommended within The Netherlands for the treatment of depression (included in the NHG/GP guidelines), it does not mean they are without side effects. While SSRI/SNRI side effects may be less severe than those associated with older antidepressant drugs, such as MAOIs and TCAs, this study suggests that they can still have a significant effect on patients' lives.

This PIP Health study found that over half of all patients using antidepressants experienced some degree of 
side effects (60\%). Any side effect has a negative effect on patients' quality of life and multiple side effects can compound and magnify the perceived severity of individual side effects. Thus, objective assessment of side-effect severity may not reflect the full extent to which side effects affect patients' daily lives.

Since the use of SSRIs became widespread, questions have arisen about their tolerability, as used in real life. Sexual dysfunction was reported in only $1.9 \%$ of patients in the prelicense, placebo-controlled clinical trials of fluoxetine, but post-marketing surveillance studies suggest rates of reported sexual dysfunction may be as high as $75 \% .^{15}$

In this real-life study of a cohort of Dutch patients with depression, the most frequently reported antidepressant side effects were 'somnolence, drowsiness, fatigue,' 'weight increase,' and 'sexual problems.' A 2001 study by Ferguson et al looking at the adverse effects associated with antidepressants yielded similar findings. Ferguson et al noted that the most troubling side effects associated with long-term SSRI therapy were sexual dysfunction, weight gain, and sleep disturbance (sleep disturbance was included as a proxy measure of patients' resulting tiredness and fatigue in the daytime). ${ }^{7}$

When asked about 'other' side effects (ie, those not included on Van Geffen's list ${ }^{12}$ ), the patients in this study produced a long and varied list of complaints (see Table 4). Although all reported side effects should be considered serious because of their impact on the daily life of the sufferer, some, such as blackouts, could be viewed as clinically more serious and potentially dangerous. The wide variety of adverse events solicited from the patients in this study highlights the importance of assessing and treating patients on a case-by-case basis and individualizing care.

Any side effect of antidepressant therapy can cause the patient to feel that their depression is not under control, which can potentially exacerbate their depressive symptoms. Discontinuation (or withdrawal) symptoms, such as depression and anxiety, are other common side effects experienced by patients who are trying to stop antidepressant therapy. The depression and anxiety associated with antidepressant withdrawal can be mistaken for a return of the original depression, often resulting in patients resuming medication, creating a vicious circle. ${ }^{17}$ Discontinuation symptoms were reported by $31 \%$ of patients in this study and assigned a high severity rating with a mean score of 7.06.

The cost of care for depression in The Netherlands is estimated at approximately 700 million euros annually. Sick leave (absenteeism) and disability due to psychological problems are substantial problems; one-third of people unable to work in The Netherlands cite 'mental disorders' as the reason they cannot work. The loss in national productivity as a result of mental health issues increases the overall cost to the economy. ${ }^{18}$ More effective management of depression could not only improve workplace productivity, but also reduce absenteeism from work. Within the PIP Health study population, almost half ( $46 \%$ ) of the patients reporting antidepressant side effects admitted to having missed work at some point as a result of their side effects. The reported impact of antidepressant therapies on patients' daily lives (summarized in Table 4) also demonstrates a loss of productivity at work. Phrases such as 'I am too tired to work,' 'too dizzy to work,' and 'I was less active both at home and at work' demonstrate the adverse effect of antidepressant therapy on some patients' productivity levels.

Far from having the desired effect of maintaining productivity and attendance at work, a significantly high level of participants using antidepressants have displayed that their work life has suffered as a result of their antidepressant treatment. A recent study conducted on behalf of Thomson Reuters evaluated the productivity-related cost of depression. Insurance claims and employee health and productivity data were analyzed for more than 34,000 patients with diagnosed depression managed on antidepressants. Significant differences were observed in terms of short-term disability (STD) and absenteeism costs between depressed patients, severely depressed patients, and the control group. The authors noted that the annual STD costs of patients with depression $(\$ 1,038)$ and severe depression $(\$ 1,685)$ were greater than those associated with other chronic diseases, such as hypertension (\$66), diabetes (\$118), chronic obstructive pulmonary disease (\$197), and rheumatoid arthritis (\$851). ${ }^{19}$ The study concluded that even when depressed patients are treated with antidepressants, the condition is associated with substantial productivity losses. ${ }^{20}$ Antidepressant therapies that offer greater symptom relief, with an improved safety and tolerability profile, could result in substantial employer savings. These conclusions are in agreement with the substantial levels of absenteeism and productivity impairment reported by the PIP Health study cohort.

\section{Study limitations and future research needs}

This study by PIP Health was conducted in early 2009 to investigate the burden associated with the licensed antidepressants available at that time. Although not all currently licensed antidepressants are included in the study analysis, 
it could be considered a good foundation study on which future research can build. Future research, however, should take into account a number of methodological findings that became apparent during the performance of this study in order to enhance the validity and reliability of the findings. This study, for example, did not differentiate between those patients with/without co-morbidities. As a result, the treatment-related side effects reported by patients receiving medication for other conditions may have biased the perceived severity and impact of their antidepressant side effects on their daily lives, as well as the time to perceived treatment benefit.

The questionnaire also failed to detail during which part of their working life and to which extent patients missed work both quantatively as well as qualitatively. Future studies evaluating the impact of antidepressant therapy on patients' work attendance and productivity should first explore in detail employment status and level, so it is possible to explore the relationship between particular side effects, lost productivity, and rates of absenteeism. These data could help to inform the health modeling of the economic impact of antidepressant therapy. The main aim of the study was to evaluate the burden of licensed antidepressant therapies, but, if this study were repeated, it would be interesting to explore in more detail the effects of antidepressants on productivity, absenteeism, and daily functioning. To do so, it would be beneficial to calculate a rate of absenteeism (rather than a figure) to provide an indication of the frequency of sick days per patient and also to compare rates of absenteeism pre- and post-initiation of antidepressant therapy.

It would also have been beneficial to capture data on the length of time that patients had been using their current antidepressant; the study placed no restrictions on treatment duration and patients could have been on the therapy for months, or only a matter of days. If duration of therapy information were available, it would be possible to assess whether there were any differences in side effects experienced by patients on short- versus long-term therapy and to evaluate whether there may be an association between side-effect burden and drug exposure.

Real-life data on antidepressant side effects remain limited. This study captured patient-reported side effects additional to those included on standard side-effects lists (eg, the Van Geffen list ${ }^{12}$ ). Thus, it could be interesting to carry out a similar study using a broader list of recognized side effects in order to assess the prevalence and impact of a wider range of side effects experienced by patients with depression.

\section{Conclusion}

Therapeutic management of depression has greatly improved in recent years, yet concerns persist around the frequency, extent, and severity of treatment-related side effects and their impact on the professional and personal lives of patients living with depression. Although antidepressants have good efficacy data, when used in real life, the associated side effects appear to be more prevalent than the early trial data would suggest. More effective management of depression requires the introduction of antidepressant therapies with efficacy profiles comparable (or better) than those of the licensed therapies included in this study, but with more favorable safety and tolerability profiles. Also important in minimizing depression relapse and antidepressant dependence will be the development of new therapies resulting in reduced discontinuation symptoms.

Patients being managed on antidepressant therapies report a wide range of side effects. Optimal therapeutic management of depression requires an individual approach to patient assessment and management (including appropriate therapy selection, counseling, lifestyle measures, and psychotherapy) rather than a 'one size fits all' approach. Until antidepressants are available that offer good efficacy and improved tolerability profiles, serious consideration should be given to the appropriateness of prescribing alternative antidepressant therapies in patients who have experienced side effects on previous antidepressants. Similarly, the pros and cons of antidepressant therapy should be considered, and discussed with patients, before prescribing decisions are made.

Further work in this area would be valuable to investigate whether the side effects of antidepressant affect patients' compliance with their prescribed regimen.

\section{Disclosure}

This work was supported by a grant from Servier Netherlands. $\mathrm{NvD}$ has received a consulting fee and research funding from Servier Netherlands.

\section{References}

1. World Health Organization (WHO) website. Mental Health: DepressionWhat is depression? Available from: http://www.who.int/mental_health/ management/depression/definition/en/index.html. Accessed June 10, 2012.

2. Murray CJ, Lopez AD. Alternative projections of mortality and disability by cause 1990-2020: Global Burden of Disease Study. Lancet. 1997;349: 1498-1504.

3. ScienceDaily (data provided by BioMed Central). Global Depression Statistics. July 25, 2011. Available from: http://www.sciencedaily.com/ releases/2011/07/110725202240.htm. Accessed May 26, 2012.

4. Hickie IB, Rogers NL. Novel melatonin-based therapies: potential advances in the treatment of major depression. Lancet. 2011;378: $621-631$ 
5. Hickie I. Is depression overdiagnosed? No. BMJ. 2007;335:329.

6. Hall WD, Mant A, Mitchell PB, et al. Association between antidepressant prescribing and suicide in Australia, 1991-2000: trend analysis. BMJ. 2003;326:1008-1010.

7. Ferguson JM. SSRI antidepressant medications: adverse effects and tolerability. Prim Care Companion J Clin Psychiatry. 2001;3:22-27.

8. Anderson IM. SSRIs versus tricyclic antidepressants in depressed patients: a meta-analysis of efficacy and tolerability. Depress Anxiety. 1998;7(Suppl 1):11-17.

9. Edwards JG, Anderson I. Systemic review and guide to selection of selective serotonin reuptake inhibitors. Drugs. 1999;57:507-533.

10. Feighner JP. Mechanism of action of antidepressant medications. J Clin Psychiatry. 1999;60(Suppl 4):4-11.

11. World Health Organization. International Monitoring of Adverse Reactions to Drugs. Adverse Reaction Terminology. Uppsala, Sweden: WHO Collaborating Centre for International Drug Monitoring; 2001.

12. Van Geffen ECG. Evaluation of patients' experiences with antidepressants reported by means of a medicine reporting system. Eur J Clin Pharmacol. 2007;63:1193-1199.

13. Dutch College of General Practitioners (NHG) website. Available from: http://nhg.artsennet.nl. Accessed June 10, 2012.

14. Baker CB, Johnsrud MT, Crismon ML, et al. Quantitative analysis of sponsorship bias in economic studies of antidepressants. $\mathrm{Br} \mathrm{J}$ Psychiatry. 2003;183:498-506.
15. Rosen RC, Lane RG, Menza M. Effects of SSRIs on sexual function: a critical review. J Clin Psychopharmacol. 1999;19:67-85.

16. Wilkinson TJ, Begg EJ, Winter AC, et al. Incidence and risk factors for hyponatraemia following treatment with fluoxetine or paroxetine in elderly people. Br J Clin Pharmacol. 1999;47:211-217.

17. Helpguide.org website. Antidepressants: What you need to know about depression medication. Available from: http://helpguide.org/mental/ medications_depression.htm. Accessed June 10, 2012.

18. Meijer SA, Smit F, Schoemaker CG, Cuijpers P, on behalf of the Rijksinstituut voor Volksgezondheid en Milieu (RIVM). Gezond verstand, Evidence-based preventie van psychische stoornissen. Available from: http://www.rivm.nl/bibliotheek/rapporten/270672001.pdf. Accessed June 10, 2012. Dutch.

19. Koster K. Study finds antidepressants provide no cure for absenteeism, 2010. Available from: http://ebn.benefitnews.com/news/antidepressantsprovide-no-cure-for-absenteeism-2682969-1.html. Accessed June 10, 2012.

20. Curkendall S. Productivity losses among treated depressed patients relative to healthy controls. J Occup Environ Med. 2010;52: $125-130$.
Patient Intelligence

\section{Publish your work in this journal}

Patient Intelligence is an international, peer-reviewed, open access journal that characterizes and measures the central role of patient behavior and intention in optimizing healthcare management in all areas of disease and complaint types. An improved understanding of patient intelligence coupled with predictive analysis helps an organization contribute more effectively to achieving better outcomes.

Submit your manuscript here: http://www.dovepress.com/patient-intelligence-journal

\section{Dovepress}

The journal is characterized by the rapid reporting of reviews, original research, methodologies, analytics, modeling, clinical studies and patient surveys across all disease areas. The manuscript management system is completely online and includes a very quick and fair peer-review system. Visit http://www.dovepress.com/ testimonials.php to read real quotes from published authors. 